\title{
Integrated cryoEM imaging center at Seoul National University, Korea
}

\author{
Miji Jeon ${ }^{\dagger}$, Jun Bae Park ${ }^{\dagger}$, Hansol Lee, Gyuhee Kim, Eung Baek Kim, Mingyu Jung and Soung-Hun Roh* \\ School of Biological Science, Institute of Molecular Biology and Genetics, Seoul National University, Seoul 08826, Republic of Korea \\ ${ }^{*}$ Correspondence: shroh@snu.ac.kr \\ ${ }^{\dagger}$ These authors contributed equally to this work.
}

The Center for Macromolecular and Cell Imaging (CMCl) is a core facility equipped with multiple cryo-capable electron microscopes including a $200 \mathrm{kV}$ Thermofisher Glacios, $200 \mathrm{kV}$ JEOL JEM2100Plus and $120 \mathrm{kV}$ FEI TECNAI with direct electron detector technologies. The center also has auxiliary equipment for optimal specimen preparation, such as for protein purification, tissue culture, grid preparation and vitrification. The center offers to the scientific community the best practice of cutting-edge cryogenic electron microscopy (cryoEM) technology for specimen optimization, highresolution data collection and structure determination. The center also develops the strategy of an integrated cryoEM imaging platform to visualize molecular architectures from purified proteins to cells.

\section{INTRODUCTION}

Over the last 10 years, cryoEM has become a powerful method to determine the structure of macromolecules and cell imaging in situ, which is the limitation of X-ray crystallography (Renaud et al., 2018). In 2017, the Nobel Prize for chemistry was awarded to Jacques Dubochet, Joachim Frank and Richard Henderson for developing cryoEM for the high-resolution structure determination of macrobiomolecules in solution. The $\mathrm{CMCl}$ was established in Korea last year to create a pipeline for systematic cryoEM research. The $\mathrm{CMCl}$ has a long-term goal of a cryoEM field basic and convergence research foundation for macromolecule and cell imaging in situ. In this report, we describe the $\mathrm{CMCl}$ with a pipeline for cryoEM and what to expect from the $\mathrm{CMCl}$ in the near future.

\section{Overall instruments in the $\mathrm{CMCl}$}

From sample preparation to three-dimensional (3D) image analysis of the protein, all of these can be resolved at the $\mathrm{CMCl}$, which is equipped with protein sample preparation for the macromolecular imaging pipeline (Figure 1A). This pipeline will lead to a leap in the development of structural biology. The $\mathrm{CMCl}$ has the equipment necessary for macromolecule structural analysis. For sample preparation, the center is equipped with two grid preparation systems, the Vitrobot Mark IV (Thermofisher, U.S.A.) and EM GP2 (Leica, Germany), and two grid charging systems, glow discharge cleaning system (Ted Pella. Inc, U.S.A.) and Tergeo plasma cleaner (PIE Scientific, U.S.A.) (Figure 1B). The $\mathrm{CMCl}$ has three transmission electron microscopes installed with additional one in planning: a 120 kV Tecnai G2 Spirit (FEI, U.S.A.), 200 kV Jeol 2100 Plus (Jeol, Japan) and $200 \mathrm{kV}$ Glacios with a Falcon 4 detector and a (FEI, U.S.A.) (Figure 1C). The Tecnai G2 Spirit and Jeol 2100 Plus are applied to macromolecular and single cell component screening before the determination of high-resolution 3D macromolecular structure using high-end microscopes. Recently, the $\mathrm{CMCl}$ installed the $200 \mathrm{kV}$ Glacios with a Falcon 4 detector (Thermofisher, U.S.A.) and a Volta phase plate. This machine is able to provide the data collection conditions desired by the user. Therefore, the Glacios has a middle-end microscope for single particle analysis (SPA) and cryo-electron tomography (cryoET). The Glacios is used for initial screening of sample quality before transferring to a $300 \mathrm{kV}$ high-end EM, which plans to be installed in the future for high-resolution data acquisition.

Advantages of $\mathbf{2 0 0}$ kV Glacios cryoEM with Falcon $\mathbf{4}$ detector Nowadays, the enhanced optical systems, electronic detectors and image processing software of cryoEM have become a key technology to study the molecular structure and mechanisms of biological macromolecules. In particular, the most important contribution to achieving atomic resolution was the improvement of the sensitivity of the direct electron detector and its operation in electron counting mode. In the $\mathrm{CMCl}$, the $200 \mathrm{kV}$ Glacios cryoEM with a Falcon 4 detector was recently set up and operated for the structural analysis of macromolecules. Its detailed specifications are documented in Table 1. Compared to the previous Falcon 3 Detector, the Falcon 4 Detector has a significantly increased internal frame rate of $250 \mathrm{fps}$ with improved detector quantum efficiency (DQE) over the entire spatial frequency range. The Falcon 4 Detector's exposure times are almost 10 times shorter than its predecessor, with an improved internal frame rate and pixel design to reduce the time to acquire the same number of images. With this ultimate DQE, microscope productivity can be greatly enhanced (Figure 2). In addition to the high quality of $\mathrm{DQE}$, the Falcon 4 detector adopts lossless data compression 


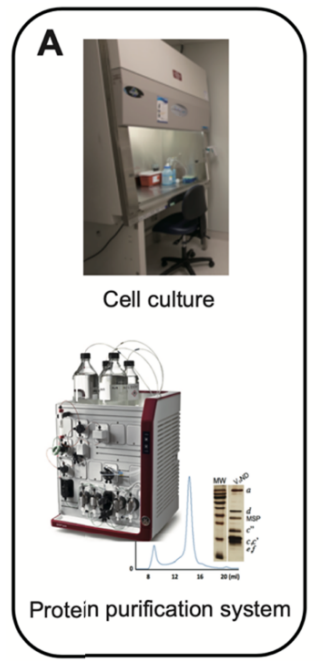

Sample preparation

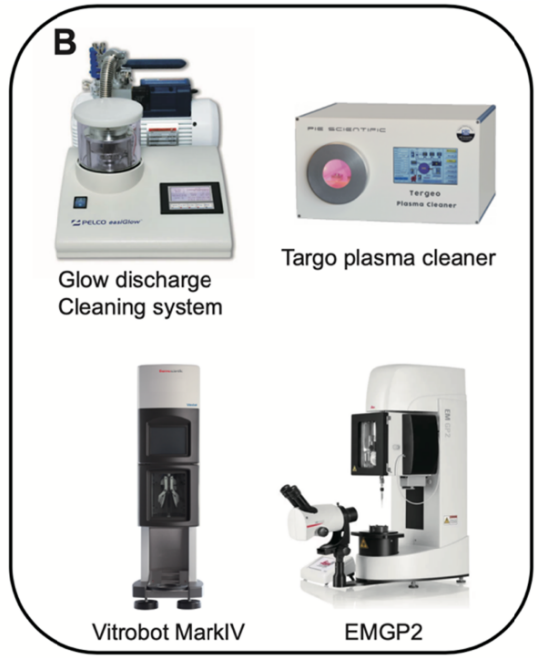

Glow discharge/Vitrification system

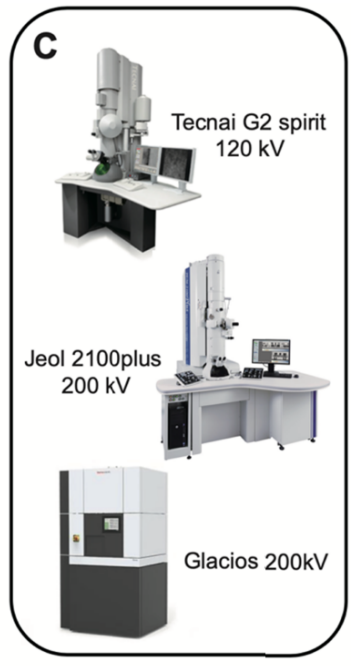

Microscope
FIGURE 1 I Core instruments in the $\mathrm{CMCl}$. Our facility established a onestop macromolecular imaging system pipeline. (A) Sample preparation pipeline, (B) cryo grid preparation system, and $(\mathrm{C})$ microscope.
TABLE 1 I Major instrumental qualifications of Glacios cryoEM with Falcon 4 detector

\begin{tabular}{|c|c|}
\hline Acceleration voltage & $80-200 \mathrm{kV}$ \\
\hline Electron source & X-FEG electron gun \\
\hline Information limit & $1.4 \AA$ \\
\hline Magnification range & $\times 25-\times 650,000$ \\
\hline Specimen tilt range & $\alpha \pm 70^{\circ}$, rotation-free imaging \\
\hline Pole piece gap & $11 \mathrm{~mm}$ \\
\hline Spherical aberration & $2.7 \mathrm{~mm}$ \\
\hline $\begin{array}{l}\text { Imaging performance } \\
\text { (Counting) }\end{array}$ & $\begin{array}{l}\operatorname{DQE}(0)=0.85 \\
\operatorname{DQE}(0.5 \mathrm{Nq})=0.60 \\
\operatorname{DQE}(1.0 \mathrm{Nq})=0.20\end{array}$ \\
\hline Chromatic aberration & $2.7 \mathrm{~mm}$ \\
\hline Detectors & $\begin{array}{l}\text { (1) 4,096 × 4,096 pixel CCD camera, for } \\
\text { specimen screening } \\
\text { (Falcon 4, Thermo Fisher Scientific Inc., USA) } \\
\text { (2) 4,096 } \text { 4,096 pixel CCD camera, for } \\
\text { specimen screening } \\
\text { (Ceta } \\
\text { (M } 16 \text { M CMOS, FEl Inc., USA) }\end{array}$ \\
\hline Dedicated software & $\begin{array}{l}\text { (1) Talos, general image display } \\
\text { (2) FluCam viewer, for image plate control and display } \\
\text { (3) EPU, for minimum dose system for automated } \\
\text { data acquisition } \\
\text { (4) EPU-D, for micro-ED processing } \\
\text { (5) FEI Tomography, for tomography data acquisition }\end{array}$ \\
\hline
\end{tabular}

using electron event representation (EER) technology. EER compression allows super resolution and avoids the need for fractioning, retaining full spatial and temporal resolution (Guo et al. 2020). To minimize damage to the cryo-sample grid during manipulation, an automated mounting system for multiple specimen grids was adopted. Different from the typical side-entry holder systems, the automated mounting system assembles specific cartridges for clipping the specimen grids. This clipping process can prevent shearing or wrinkling of the thin cryo grids.
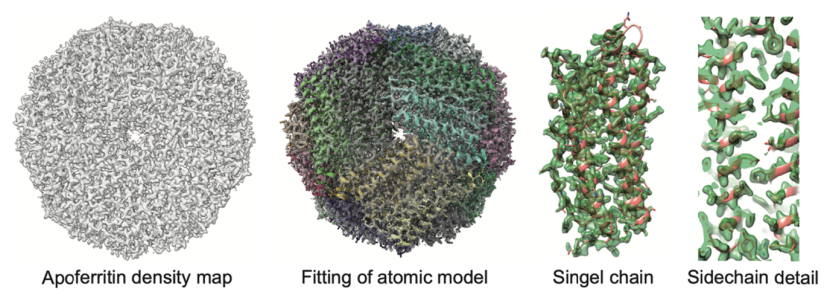

FIGURE 2 I 2.1 A cryoEM structure of horse spleen apoferritin. CryoEM density map, fitting of atomic model, single chain, and sidechain detail of horse spleen apoferritin using the Glacios $200 \mathrm{kV}$ EM device.

The clipped cryo grids are loaded into a cassette that can hold up to 12 cartridges. The cassettes are then docked into the contamination-free auto-loader where the grid is kept under vacuum at liquid nitrogen temperature. Inside the Auto-loader system, grids can be freely mounted and unmounted, and their ice quality can be automatically examined by Atlas software in EPU. This EPU system not only allows users to select squares and holes of proper ice thickness, but also to easily collect the whole cryoEM dataset with minimized damage to the specimen through the EPU interface. Users can conveniently transfer the collected images through our established server system as we will describe further.

\section{Single-particle cryoEM workflow}

The $\mathrm{CMCl}$ has a one-stop pipeline for single particle analysis (SPA). The SPA workflow is classified into six steps (Figure 3). For single-particle analysis, we have to preferentially prepare high-quality protein samples. Generally, protein preparation is done using immobilized affinity chromatography (IMAC), ion-exchange chromatography (IEX) and size-exclusion chromatography (SEC). To check the protein quality status for single-particle analysis, negative staining is a suitable method. Negative staining can qualitatively evaluate particle distribution 

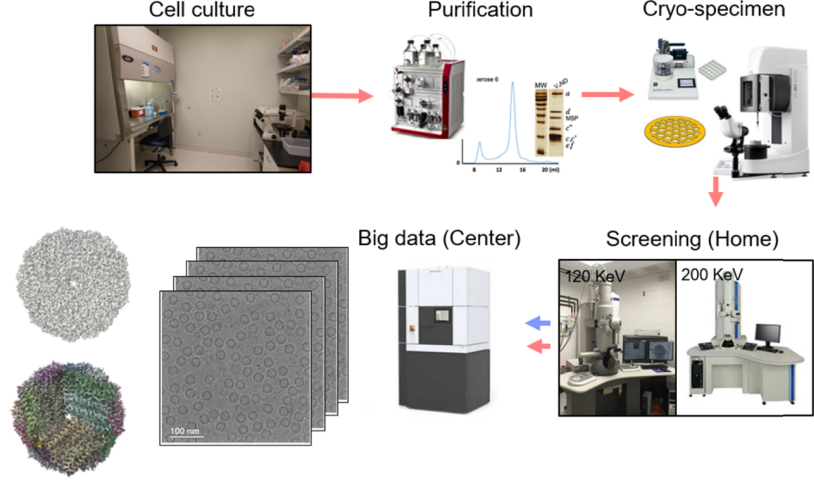

FIGURE 3 I Single particle analysis (SPA) workflow. SPA analysis workflow in the $\mathrm{CMCl}$. The $\mathrm{CMCl}$ has a single particle analysis pipeline in SNU. Our center established a One-step single-particle analysis (SPA) pipeline. According to the cryo-EM field test using horse spleen apoferritin, we got high-resolution $(2.1 \AA)$ apoferritin structure.

and arrangement. After checking the particle contribution and arrangement, we have to prepare a vitrified sample of the particle in its native state. Then the sample quality is evaluated using a cryoEM (i.e., Jeol-200 plus, Glacios). Checking a suitable cryo sample grid using a cryoEM, they conduct large data collection acquisition and 3D model reconstruction for high-resolution maps.

CryoET, a powerful method for identifying 3D structures in situ The cryoEM method has been used to identify the 3D structures of macromolecules, including dynamic molecular complexes, in near-native conditions at close to atomic resolution (Murata and Wolf, 2018). However, cryoEM has limitations in visualizing a targeted structure in situ. CryoET is a powerful method that can provide 3D views of a single particle or macromolecules in cells in a near-native frozen-hydrated state from a series of twodimensional tilted images ( $\mathrm{Ng}$ and Gan, 2020). Furthermore, it is important to have an appropriate thickness of a biological sample for a high-quality image in a cryoET study. A suitable computational technique is also key to collect and analyze a series of images to reconstruct the 3D structure of the target (Doerr, 2017). In our cryoET study, the Serial EM, which is able to operate an automatic series of tilted images, was installed in our JEOL $200 \mathrm{kV}$ microscope to collect tilted images of the target. We are also setting up cryo-correlative light and electron microscopy (cryoCLEM) including a cryo-focused ion beam (cryoFIB) combined with a scanning electron microscope (SEM). The overall workflow of the cryoET is shown below, in which several images of the sample are taken at different tilt angles and the images are stacked and processed for 3D reconstruction (Figure 4).

\section{Automated tomography in EM: Serial EM}

Serial EM is an automated program that measures and collects data of the tilt series of the sample based on the coordinates

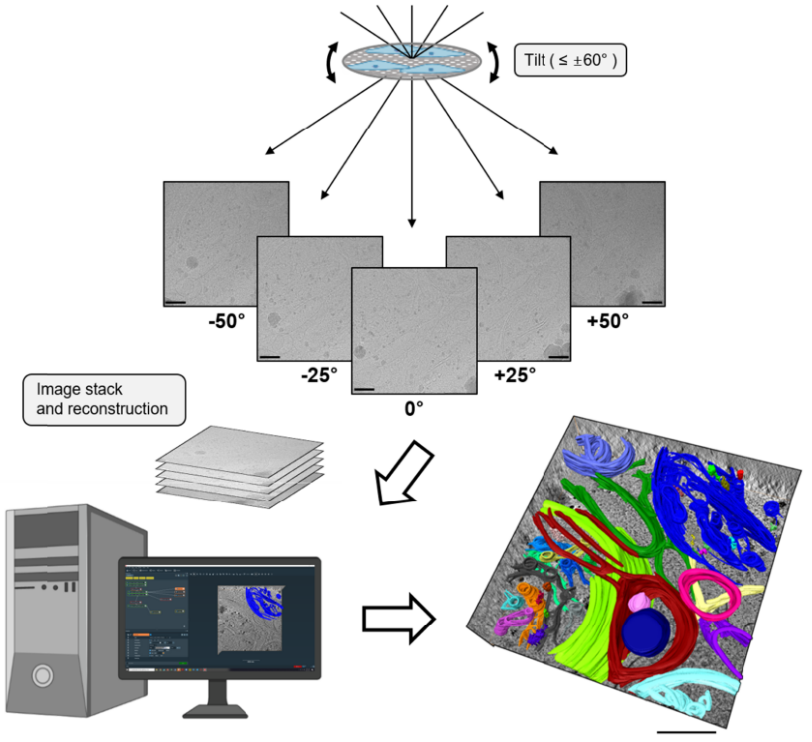

FIGURE 4 I Overview of cryoET workflow. CryoET data will be collected from a series of tilting images via Glacios and then reconstructed as an image stack via Amira to visualize 3D cellular structural modeling (HeLa cells). Scale bar is $200 \mathrm{~nm}$.

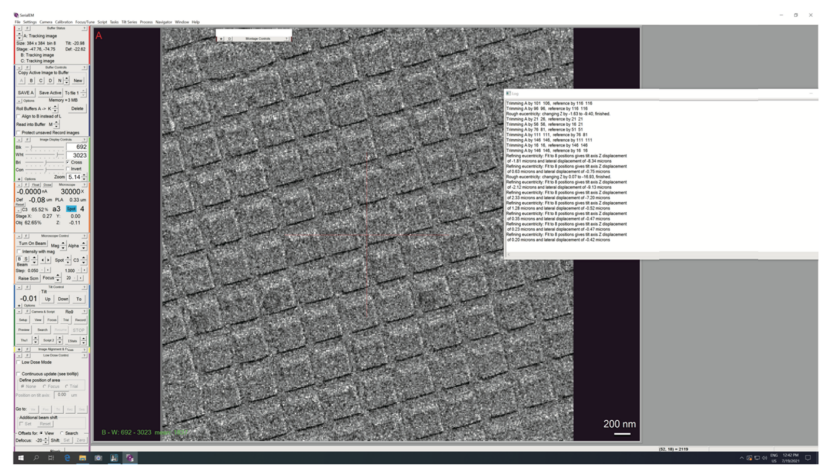

FIGURE 5 I Serial EM interface shown with the calibration grid (Ted Pella 603). The serial EM interface is shown on the left side of the figure. The current status of the tilt angle, coordinates and size of the buffer image are shown. The spot size, magnification, tilt angle and coordinates of the buffer image can be changed.

of the previous tilt angle. The manual operation of collecting data of the tilt series is tedious and time-consuming, so the Serial EM alleviates these problems. The Serial EM can be implemented in Tecnai or JEOL EM for tomography (Mastronarde, 2005). The $\mathrm{CMCl}$ is the first in South Korea to implement the Serial EM on JEOL $200 \mathrm{kV}$ focusing on cellular tomography, which takes 2D images of the sections of target organelles inside the vitrified cellular samples by tilting them at $\pm 60^{\circ}$ angles to obtain the 3D volumes (McDonald and Auer, 2006; Hoenger and Bouchet-Marquis, 2011). Furthermore, this program uses cross correlation between two different images and autofocusing based on positive and negative beam tilt for 
image acquisition and data collection (Mastronarde, 2005). As shown in Figure 5, the serial EM interface shows the current status of the image and components that can be manipulated to obtain a higher image resolution. Using the Serial EM, we are anticipating collecting in situ cellular structures through imaging. As mentioned before, cellular tomography can be done using Glacios, but the Serial EM in JEOL can be used as well.

Mechanism and pipeline for in situ macromolecule imaging using cryoCLEM

CryoCLEM is a combination of fluorescence microscopy and electron microscopy. Due to the macromolecules inside cells, it has been challenging to obtain an image of a target structure with cryoEM. For effective visualizing, cryo-fluorescence microscopy can be helpful to find the coordinates of the targets, and then the information can be transferred to EM (Kuba et al., 2021). In this way, the macromolecules in the cells can be imaged and many biological phenomena in the cells can be analyzed (Figure 6).

A considerable limitation of cellular tomography is the thickness of the samples. A cell must be at a certain thickness through the milling process for the image of the cell to be taken at a good resolution. The milling process reduces the number of molecules inside the cell, which influences the imaging process. Therefore, a milling process using cryoFIB is needed, followed by monitoring the sample with SEM. With cryoFIB/ SEM, samples become thinner until the thickness is $0.2-20$ um. Moreover, cryo-FIB/SEM combined with fluorescence microscopy can be a powerful method: the location of targets can be identified by fluorescence microscopy, and then FIB can mill samples around the targets' position. Then lamellae will be formed and targets are easily visualized by using cryoET (Kuba et al., 2021). The $\mathrm{CMCl}$ purchased cryoFIB/SEM equipment, the Aquilos2 FIB/SEM (Thermofisher) and is going to install it this year. After this pretreatment, the targets are imaged by the $200 \mathrm{kV}$ EM Glacios. Three-dimensional structures are reconstructed using the analytic software RELION/EMAN2. The high-resolution structures of macrobiomolecules in cells are anticipated through this equipment.

\section{Computing power for cryoEM image processing}

For the high-resolution reconstruction of biological samples, large amounts of movie files are necessarily collected with a transmission electron microscope. The raw data, in a single particle analysis case, is then processed to reconstruct a 3D structure of the protein of interest from the two-dimensional images. A high level of computer performance is required in this process, and for this reason, high computer performance is one of the important factors in the 3D structure reconstruction process using cryoEM.

CryoSPARC (Punjani et al., 2017) and RELION (Zivanov et al., 2018), which are mainly used for data processing, have provided computer specifications to run the software on the website (CryoSPARC: https://guide.cryosparc.com; RELION: https://www3.mrc-Imb.cam.ac.uk/relion), and the characteristic fact here is that a GPU-based processor is used to efficiently and quickly analyze a huge amount of data. A cheaper and more accessible CPU-based software has been upgraded to enable operation (Biyani et al., 2017; Zivanov et al., 2018), but a more powerful GPU-based processor is still essential to analyze a large number of data sets in parallel. In addition, in order to attempt simultaneous analysis of already developed or additionally developed data processing software, a device with higher performance is required even for the same GPU-based machine. One of the built servers has DAS format, and the server and storage are connected at the SAS $12 \mathrm{G}$ level. The others transfer the data through a $10 \mathrm{G}$ network switch.

The detailed description of the computer power provided in the $\mathrm{CMCl}$ is specified in Table 2. Based on the server alone, the CPU and GPU clusters show $1.72 \times 10$ and $3.34 \times 10^{2}$ Tflops, respectively, and about 744 TB is installed as a storage space for raw data and for data processing. The user can access the equipment at the $\mathrm{CMCl}$ through the collaboration with the center. The local computers are limited for data confirmation

\section{Sample preparation}

A

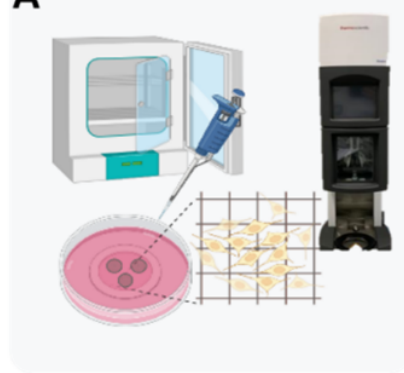

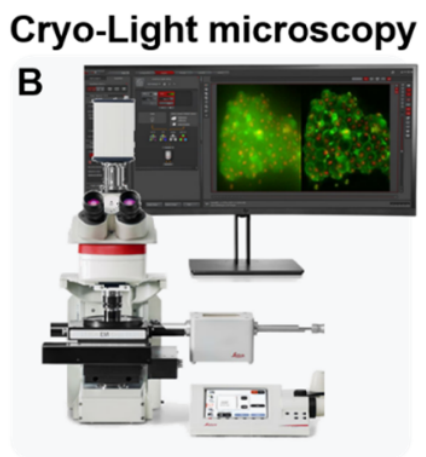

Cryo-FIB/SEM

C

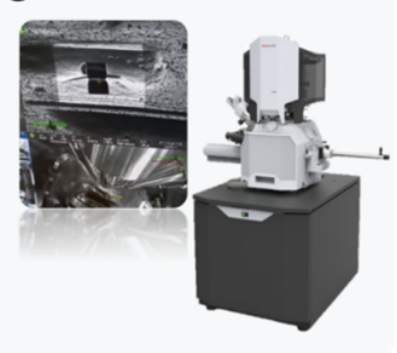

Cryo-EM

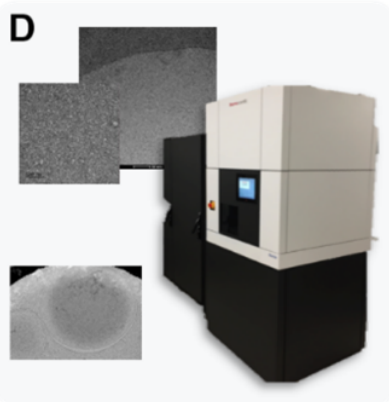

FIGURE 6 I CryoCLEM workflow in the CMCI. (A) Sample preparation: An in situ sample is prepared by the Vitrobot Mark IV (Thermofisher Scientific). (B) Cryo-light microscopy: A target labeled with fluorescent tags is imaged by cryo-light microscopy (Leica microsystems) to identify the exact location in near-native conditions. (C) Cryo-FIB/SEM (Thermofisher Scientific): Using cryo-FIB, the thickness of the sample can be controlled. (D) Cryo-EM/ET: The prepared sample (HeLa cells) is finally imaged with Glacios (Thermofisher scientific) along with cryo-EM/ET. 
TABLE 2 I Specifications of high-performance computers and servers in the $\mathrm{CMCl}$

\begin{tabular}{|c|c|c|c|c|c|c|}
\hline Machine & Device & Detail & EA & Total & Unit & $\begin{array}{l}\text { Perform- } \\
\text { ance } \\
\text { (Tflops) }\end{array}$ \\
\hline \multirow{4}{*}{ GPU Server 1} & Server & Dell T640 Server & & & & \\
\hline & Processor & $\begin{array}{l}\text { Intel }^{\circledR} \text { Xeon }^{\top \mathrm{M}} \text { Processor Gold } 6130 \\
(2.1 \mathrm{GHz}, 22 \mathrm{MB} \text { Cache, QPI 10.40GT/sec, 16-Core) }\end{array}$ & 2 & 32 & Core & 2.15 \\
\hline & SSD & 480GB SSD Drive (OS Mirror) & 2 & 960 & GB & \\
\hline & GPU & nVidia Titan Xp 3840Core/1582Mhz/12GB GDDR5X & 4 & 48 & GB & 48.4 \\
\hline \multirow{6}{*}{ GPU Server 2} & Server & CryoServer208 8GPU Server & & & & \\
\hline & Processor & $\begin{array}{l}\text { Intel }^{\oplus} \text { Xeon }^{\mathrm{TM}} \text { Processor Gold } 6242 \mathrm{R} \\
(3.1 \mathrm{GHz}, 22 \mathrm{MB} \text { Cache, 20-Core) }\end{array}$ & 2 & 40 & Core & 3.96 \\
\hline & RAM & 16GB RDIMM, 2666MT/s, Dual rank & 12 & 192 & GB & \\
\hline & SSD & 512 MLC SSD Drive (O/S Mirror) & 2 & 1024 & GB & \\
\hline & SSD & 1TB TLC 2.5" SSD Drvie (Raid 5-Scratch) & 6 & 6 & TB & \\
\hline & GPU & NVIDIA RTX 3090 GDDR6X 24GB GPU Card & 8 & 192 & GB & 284 \\
\hline \multirow{5}{*}{ Cluster Master Node } & Server & Hpclab Server System Cryo-R221-X08I & & & & \\
\hline & Processor & $\begin{array}{l}\text { Intel }^{\oplus} \text { Xeon }^{\mathrm{TM}} \text { Processor Silver 4210R } \\
(2.4 \mathrm{GHz}, 13.75 \mathrm{MB} \text { Cache, 10-Core) }\end{array}$ & 2 & 20 & Core & 1.5 \\
\hline & RAM & 16GB RDIMM, 2933MT/s, Dual rank & 12 & 192 & GB & \\
\hline & SSD & 480GB SSD Drive (Mirror) & 2 & 960 & GB & \\
\hline & HDD & $\begin{array}{l}\text { 10TB 7.2K RPM NLSAS 12Gbps 512n 3.5" } \\
\text { Hot-plug Drive (Raid 6) }\end{array}$ & 6 & 60 & TB & \\
\hline \multirow{4}{*}{ Cluster Computing Node } & Server & Hpclab Server System Cryo-R121-X04I & & & & \\
\hline & Processor & $\begin{array}{l}\text { Intel }{ }^{\circledR} \text { Xeon }^{\text {TM }} \text { Processor Gold 6240R } \\
(2.4 \mathrm{GHz}, 35.75 \mathrm{MB} \text { Cache, 24-Core 48-Thread) }\end{array}$ & 2 & 48 & Core & 3.68 \\
\hline & RAM & DDR4-2933Mhz 16GB DDR4 Reg.ECC SDRAM & 12 & 192 & GB & \\
\hline & SSD & 480GB SSD 6Gbps OS Drive & 1 & 480 & GB & \\
\hline \multirow{4}{*}{ Cluster Computing Node (2 EA) } & Server & Hpclab Server System Cryo-R121-X04I & & & & \\
\hline & Processor & $\begin{array}{l}\text { Inte }^{\oplus} \text { Xeon }^{\mathrm{TM}} \text { Processor Gold 6240R } \\
(2.4 \mathrm{GHz}, 35.75 \mathrm{MB} \text { Cache, 24-Core 48-Thread) }\end{array}$ & 4 & 96 & Core & 7.37 \\
\hline & RAM & 16GB RDIMM, 2933MT/s, Dual rank & 12 & 192 & GB & \\
\hline & SSD & 480GB SSD 6Gbps OS Drive & 1 & 480 & GB & \\
\hline \multirow{5}{*}{ Data storage } & 8TB HDD & Dell MD1400 Storage (Physical-96TB) & 12 & 96 & TB & \\
\hline & 10TB HDD & Dell MD1400 Storage (Physical-120TB) & 12 & 120 & TB & \\
\hline & 12TB HDD & Dell MD1400 Storage (Physical-144TB) & 12 & 144 & TB & \\
\hline & 16TB HDD & Dell MD1400 Storage (Physical-192TB) & 12 & 192 & TB & \\
\hline & 16TB HDD & Dell MD1400 Storage (Physical-192TB) & 12 & 192 & TB & \\
\hline \multirow{4}{*}{ Desktop 1 (2 EA) } & Processor & Intel Core9 i7-9700K Processor & & & & \\
\hline & RAM & DDR4 16GB * 2EA & 2 & 32 & GB & \\
\hline & $\mathrm{HDD}$ & SATA 4TB HDD & 1 & 4 & TB & \\
\hline & GPU & NVIDIA Geforce RTX 2070 GPU CARD & 2 & & & \\
\hline \multirow{4}{*}{ Desktop 2 (2 EA) } & Processor & Intel Core 10 i7-10700 /2.9Ghz/16MB/8core & & & & \\
\hline & RAM & DDR4 16GB & 2 & 32 & GB & \\
\hline & HDD & SATA 8TB HDD & 1 & 8 & TB & \\
\hline & GPU & $\begin{array}{l}\text { Galaxy Geforce RTX } 2070 \text { SUPER EX WHITE } \\
\text { OC D6 8GB }\end{array}$ & 2 & & & \\
\hline
\end{tabular}

and simple data analysis. If there are problems due to the data storage limitation, it is also possible to use KISTI highperformance computer storage space for data analysis. In the future, according to the increasing number of projects and users, the $\mathrm{CMCl}$ has plans to continuously increase the computer specifications including the data processor and storage space. 


\section{CONCLUSION}

The equipment provided in the $\mathrm{CMCl}$ introduced above can be used for research purposes. However, the equipment for cell culture and protein purification must be used under supervision. In order to use the equipment, the users are required to make a reservation in advance on the ZEUS website (https://www. zeus.go.kr/cloud/cmci). It is possible to check the available time and equipment expenses, as well as information on available same model equipment in other organizations. In the case of consumables, the users need to additionally purchase the proper amount of items. Before making a reservation, it is highly recommended to consult a management in charge to confirm the availability of consumables. CryoEM itself, the cameras used in microscopes, electron detectors, and computer programs for data processing, will continue to evolve in the future. These future technologies will overcome the limitations of current imaging techniques for biological specimens and also keep being improved in the aspect of user accessibility. The $\mathrm{CMCl}$ will contribute to elucidating important biological questions while continually updating newly developed technologies and supplementing the appropriate equipment.

\section{ACKNOWLEDGEMENTS}

S.-H.R. acknowledge the financial support from CreativePioneering Researchers Program through Seoul National University and the NRF grant funded by the Korean government (NRF2019M3E5D6063871, 2019R1C1C1004598, 2020R1A5A1018081, 2021M3A9I4021220 and 2020R1A6C101A183). M.J.J. (2021R1I1A1A0104038911, 2021R1A6C103B381) and G.H.K. (2021R111A1A0104928411) acknowledge the financial support from NFEC and NRF grant funded by the Korean government.

Original Submission: Sep 16, 2021

Revised Version Received: Dec 14, 2021

Accepted: Dec 21, 2021

\section{REFERENCES}

Biyani, N., Righetto, R.D., McLeod, R., Caujolle-Bert, D., Castano-Diez, D., Goldie, K.N., and Stahlberg, H. (2017). Focus: the interface between data collection and data processing in cryo-EM. J Struct Biol 198, 124133.

Doerr, A. (2017). Cryo-electron tomography. Nat Methods 14, 34.

Guo, H., Franken, E., Deng, Y., Benlekbir, S., Singla Lezcano, G., Janssen, B., Yu, L., Ripstein, Z.A., Tan, Y.Z., and Rubinstein, J.L. (2020). Electron-event representation data enable efficient cryoEM file storage with full preservation of spatial and temporal resolution. IUCrJ 7, 860869.

Hoenger, A., and Bouchet-Marquis, C. (2011). Cellular tomography. Adv Protein Chem Struct Biol 82, 67-90.

Kuba, J., Mitchels, J., Hovorka, M., Erdmann, P., Berka, L., Kirmse, R., KÖnig, J., J, D.E.B., Goetze, B., and Rigort, A. (2021). Advanced cryotomography workflow developments - correlative microscopy, milling automation and cryo-lift-out. J Microsc 281, 112-124.

Mastronarde, D.N. (2005). Automated electron microscope tomography using robust prediction of specimen movements. J Struct Biol 152, 3651.

McDonald, K.L., and Auer, M. (2006). High-pressure freezing, cellular tomography, and structural cell biology. Biotechniques 41, 137-143.

Murata, K., and Wolf, M. (2018). Cryo-electron microscopy for structural analysis of dynamic biological macromolecules. Biochim Biophys Acta Gen Subj 1862, 324-334.

Ng, C.T., and Gan, L. (2020). Investigating eukaryotic cells with cryoET. Mol Biol Cell 31, 87-100.

Punjani, A., Rubinstein, J.L., Fleet, D.J., and Brubaker, M.A. (2017). cryoSPARC: algorithms for rapid unsupervised cryo-EM structure determination. Nat Methods 14, 290-296.

Renaud, J.P., Chari, A., Ciferri, C., Liu, W.T., Rémigy, H.W., Stark, H., and Wiesmann, C. (2018). Cryo-EM in drug discovery: achievements, limitations and prospects. Nat Rev Drug Discov 17, 471-492.

Zivanov, J., Nakane, T., Forsberg, B.O., Kimanius, D., Hagen, W.J., Lindahl, E., and Scheres, S.H. (2018). New tools for automated highresolution cryo-EM structure determination in RELION-3. Elife 7, e42166. 